\title{
Effects of Salts on Rheological Behaviour of Salvia Hydrogels
}

\author{
Rike Yudianti* and Myrtha Karina \\ Research Centre for Physics, Indonesian Institute of Sciences, Bandung - Indonesia \\ Masahiro Sakamoto and Jun-ichi Azuma \\ Laboratory of Forrest Biochemistry, Division of Enviromental Science, Graduate School of Agriculture, \\ Kyoto University, Japan
}

Received August 14, 2008; Revised October 3, 2008; Accepted October 16, 2008

\begin{abstract}
Rheological behavior of natural hydrogel produced from seeds of three Salvia spp. (S. miltiorrhiza (SM), S. sclarea (SS), S.viridis (SV)) was investigated by using a Rheometer equipped with a cone and plate geometry measuring system under never-dried condition. Different chemical contents of such hydrogels give significant effects on their rheological properties. Because of incomplete penetration of water inside the hydrogels after drying before-dried hydrogels were used for rheological analysis. To know molecular interactions which predominated in the gel formation, some constituents were externally added to the $1.0 \%(\mathrm{w} / \mathrm{w})$ hydrogel. Addition of urea to disrupt hydrogen bonds reduced 3.4-67\% viscosity of the untreated hydrogels and changed viscoelastic properties from gel to liquid-like behavior. Neutral salts added to the hydrogel solution at $0.1 \mathrm{M}$ also lowered the viscosity in a manner related with increase in size of cations and temperature. Changing from gel state to liquid-like state was also easily confirmed by oscillation measurement (storage, $G^{\prime}$, and loss, $G^{\prime \prime}$, modulii) typically observed in the cases of potassium sulfate and potassium thiocyanate. Influence of $\mathrm{pH}$ variation on the viscosity explained that weak alkaline condition ( $\mathrm{pH} \mathrm{8-9)}$ creates a higher resistance to flow due to increasingly electrostatic repulsions between negative charges ( $\left.\mathrm{COO}^{-}\right)$. Importance of calcium bridges was also demonstrated by recovery of viscosity of the hydrogels by addition of calcium after acidification. The summarized results indicate that electrostatic repulsion is a major contributor for production of hydrogel structure.
\end{abstract}

Keywords: rheology, rheometer, hydrogel, salvia spp, viscosity, viscoelasticity, electrostatic repulsion.

\section{Introduction}

Lamiaceae is one of a few families including various aromatic plants that provide some of essential ingredient of life in enhancing the desirable flavor, aroma of food and drink and utilizing as a folk medicine. Salvia is one of genera in Lamiaceae family found wild in South America, Southern Europe, Northern Africa and Asia which utilized for medical, ornamental and qulinary qualities. Yudianti et al. ${ }^{1,2}$ analyzed morphological and chemical properties of hydrogels from three typical Salvias, S. miltiorrhiza (SM), S. sclarea (SS) and S. viridis (SV). Such hydrogels belong to anionic polysaccharides which have aproximately 25-30\% of uronic acid in their acidic fractions. According to the previous work, ${ }^{2,3}$ such hydrogels were identified as polysaccharide gels which contained glucuronic acid linked to xylan as dominant polysaccharide. Such hydrogels are rich in divalent cations of magnesium and calcium distributing in 0.17 (SM), 0.13

*Corresponding Author. E-mail: rikeyudianti@yahoo.com
(SS), 0.8\% (w/w) (SV) and 0.44 (SM), 0.46 (SS), 1.12 (SV) \% $(\mathrm{w} / \mathrm{w})$, respectively. These divalent metal ions have possibility of making dense cross-linkages between two carboxyl groups by ionic bonds to form three dimensional network structure as speculated in gellan gel by using computer modeling. ${ }^{4}$ This was also revealed by Loizou et al. and Torres et al. ${ }^{5,6}$ who studied cross-linking formation by addition of iron, dichromate to protein gel and glutaraldehyde to chitosan solution, respectively.

Polymer gels play a ubiquitous role in modern science and technology. It is well known that polymer gels can deform in response to certain stimuli such as $\mathrm{pH}$, temperature and electric field. Rheological measurement provides an appropriate tool to obtain information about microstructure which is useful to understand macroscopic behavior in terms of the microstructure organization. It gives also new insights about the physical mechanisms that govern the unique mechanical properties of soft materials. Rheological properties of polysaccharide gels were also studied in gellan gel, ${ }^{2,7-10}$ sodium alginate ${ }^{11}$ and carrageenan. ${ }^{12}$ Understanding 
dynamic response of hydrogel is essential for designing material having desirable performance. When strain deformation is applied on the polymer gel, stress response may modify the shape of the gel surface. Change in the surface shape will modify the dynamic fluid flow reflecting the intimate coupling between liquid and solid.

In this paper, we investigated the viscoelasticity and viscosity properties of hydrogels produced from three species of Salvias, SM, SS and SV, influenced by $\mathrm{pH}$, temperature and addition of salts including calcium. In order to explain the above mentioned rheological characteristics, it is essential to consider the various interaction forces that may exist in the molecular architecture of the hydrogels and how these are influenced by $\mathrm{pH}$ and additives. The purpose of this study is also to find out the predominant interactions that work in the hydrogels. This investigation is necessary to provide potential utilization for the hydrogels as standard experimental tools for studying the gelation kinetics of soft materials.

\section{Experimental}

Material and Preparation. Seeds of three species of Salvias (S. miltiorrhiza (SM), S. sclarea (SS), S. viridis (SV)) in Lamiaceae family used as source of hydrogels were purchased from Richters Co., Ontario, Canada. After soaking in water, hydrogels expanded out from exocarp layer of seeds were isolated by treatment of electric mixer for $7 \mathrm{~s}$. and subsequent filtration through $180 \mu \mathrm{m}$ screen. Hydrogel solution was concentrated to above $1.5 \% \mathrm{w} / \mathrm{w}$ by using vacuum evaporator to control amount of water in gel structure and freeze dried to evaluate chemical properties. Beforedried hydrogel (BDH) with concentration of $1.0 \%(\mathrm{w} / \mathrm{w})$ was obtained by dilution of the concentrated gel by addition of an appropriate amount of pure water. Similarly concentration of hydrogels was changed by controlling the amount of water.

Variation of pH on Salvia Hydrogels. Native hydrogels from three species of Salvia (SM, SS, SV) have different $\mathrm{pH}$ values of 6.2, 6.4, 7.2, respectively. Values of $\mathrm{pH}$ were adjusted to $1.0,1.9,2.3,4.3,6.2,7.2,9.2,11.2,12.3$ by using Carmody's buffer solution, concentrated hydrochloric acid and $0.5 \%$ and $7 \%(\mathrm{w} / \mathrm{v})$ sodium hydroxide solution. The hydrogels were kept in refrigerator at $4{ }^{\circ} \mathrm{C}$ for one night before measurement.

Addition of Urea on Salvia Hydrogels. Contribution of hydrogen bonds in gel formation was analzed by addition of urea to the hydrogels, since urea disrupts hydrogen bonds and changes water structure. Eight molar concentration of urea was used to achieve 3.5-4.2 M urea in 1\% (w/w) hydrogel concentration. Urea-containing hydrogels were mixed carefully at room temperature and kept in refrigerator at $4^{\circ} \mathrm{C}$ for one night before measurement.

Addition of Calcium on Salvia Hydrogels. A standard calcium solution of 1,000 ppm for elemental analysis was used as one of divalent cations to know effectiveness of calcium bridge formation in gel structure. Before addition of calcium, $\mathrm{pH}$ of each hydrogel was adjusted to acidic condition (pH 1-1.5) with addition of appropriate amount of $\mathrm{HCl}$ and fully mixed at room temperature. Calcium ions were added to the acidified gels to make $0.7 \times 10^{-3}, 1.6 \times 10^{-3}$ and $2.8 \times 10^{-3} \%(\mathrm{w} / \mathrm{w})$ in terms of added ions, mixed and kept for $2-3 \mathrm{~h}$ at room temperature. Hydrogels were then neutralized to $\mathrm{pH}$ 6.8-7.2 by addition appropriate amount of 0.5 and $7 \%$ $(\mathrm{w} / \mathrm{v}) \mathrm{NaOH}$. Subsequently pure water was added to make $1 \%(\mathrm{w} / \mathrm{w})$ hydrogel concentration, and kept at least one night in refrigerator at $4^{\circ} \mathrm{C}$.

Effects of Salt Addition on the Viscoelasticity and Viscosity of Salvia Hydrogels. Potassium thiocyanate, potasium sulfate and neutral alkali-metal salts having different ion radius ( $\mathrm{LiCl}, \mathrm{NaCl}, \mathrm{KCl}, \mathrm{RbCl}$ and $\mathrm{CsCl}$ ) were selected to analyze effects of salts on gellous properties of Salvia hydrogels. One molar of salt solution was prepared and added to each hydrogel to make $0.1 \mathrm{M}$ in $1 \%(\mathrm{w} / \mathrm{w})$ hydrogel concentration. The $\mathrm{pH}$ of each hydrogel was kept at the same level as in the native state.

Dynamic Viscoelasticity Measurement. Rheological properties of Salvia hydrogels were measured by using a Dynamic Viscoelasticity Measurement Apparatus (Reogel-G1000, UBM Co., Ltd.) equipped with cone and plate geometry of $40 \mathrm{~mm}$ diameter and $4^{\circ}$ angle. The gap distance was fixed at $50 \mu \mathrm{m}$. A small amount of silicon oil was poured into a round groove formed at the outer region of the plate to prevent evaporation of water from the hydrogels. After samples were loaded to rheometer plate, measurement was performed at 30, 40, 50 and $60^{\circ} \mathrm{C}$. The plate was rotated with applied sinusoidal shear deformation $\gamma(\mathrm{t})=\gamma(0) \sin (\omega t)$ at $0.1-10 \mathrm{~Hz}$. Simultaneously, the time dependent stress was quantified by measuring the torque given by $\tau(t)=G^{\prime}(\omega) \gamma o \sin (\omega t)+G^{\prime \prime}(\omega) \gamma o$ $\cos (\omega t)$ (where $\gamma$ indicates shear rate). First, shear ratedependent measurement of apparent viscosity were carried out in the range of 0.01 to $100 \mathrm{rad} / \mathrm{sec}$, to test the viscoelastisity of the samples. Dynamic frequency sweep test were performed in the frequency range of $0.1-10 \mathrm{~Hz}$ to record storage modulus $\left(G^{\prime}\right)$, loss modulus $\left(G^{\prime \prime}\right)$ and loss factor $(\tan \delta)$.

\section{Results and Discussion}

Viscoelasticity properties representated by storage modulus $\left(G^{\prime}\right)$, loss modulus $\left(G^{\prime \prime}\right)$ and loss factor $(\tan \delta$ ) elucidate response of hydrogel when oscillation frequency applied. Generally, all species show typical viscoelasticity and viscosity properties at the same concentration of $1.0 \%(\mathrm{w} / \mathrm{w})$. We compared the properties between before dried (BD) (Figure 1(a)) and after dried (AD) hydrogels (Figure 1(b)). As shown in Figure 1(a), all Salvia hydrogels (BD) have solidlike properties $\left(G^{\prime}>G^{\prime \prime}\right)$ and independence of dynamic modulus in response to frequency was clearly observed in SM 


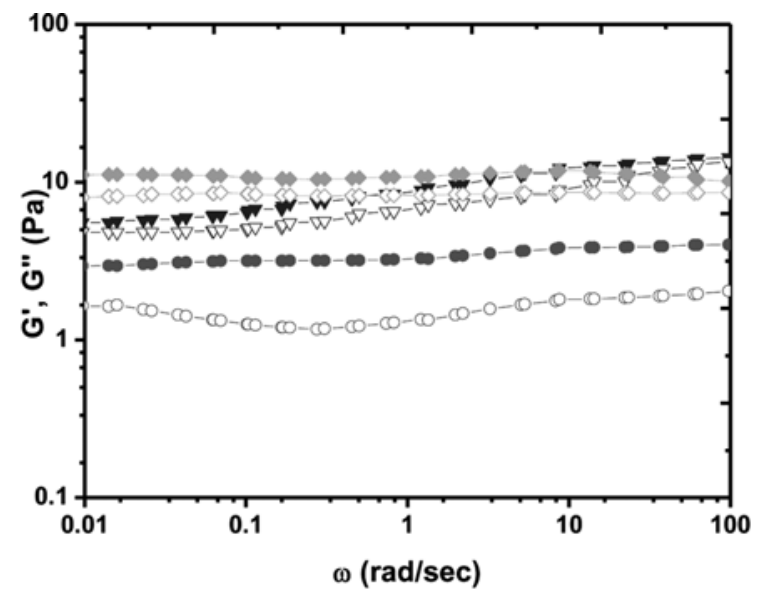

(a)

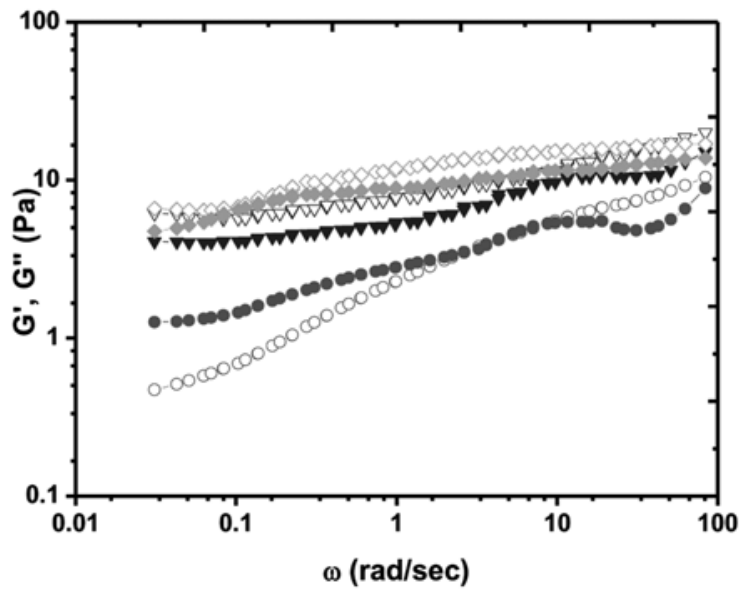

(b)

Figure 1. Viscoelasticity profiles of salvias hydrogels before and after drying. (a) BD Salvia hydrogels ( $\bullet, G$, SM-BD; $\bigcirc, G^{\prime \prime}$, SM$\left.\mathrm{BD} ; \boldsymbol{\nabla}, G^{\prime}, \mathrm{SS}-\mathrm{BD} ; \nabla, G^{\prime \prime}, \mathrm{SS}-\mathrm{BD} ; \bullet, G^{\prime}, \mathrm{SV}-\mathrm{BD} ; \diamond, G^{\prime \prime}, \mathrm{SV}-\mathrm{BD}\right)$ and (b) AD Salvia Hydrogels (,$G^{\prime}$, SM- AD; $\bigcirc, G^{\prime \prime}, \mathrm{SM}-\mathrm{AD}$; $\nabla$, $G^{\prime}$, SS- AD; $\nabla, G^{\prime \prime}$, SS- AD; $\left.\bullet, G^{\prime}, \mathrm{SV}-\mathrm{AD} ; \diamond, G^{\prime \prime}, \mathrm{SV}-\mathrm{AD}\right)$.

and SV hydrogels with the SM hydrogel having the lowest dynamic modulus. In AD gels, however, dependence in frequency range $0.1-10 \mathrm{~Hz}$ was present and dynamic modulus gradually increased with frequency and changed from solid $\left(G^{\prime}>G^{\prime \prime}\right)$ (BD) to liquid-like $\left(G^{\prime}<G^{\prime \prime}\right)$ (AD) properties. These differences in viscoelastic properties of Salvia hydrogels at the same concentration (1.0\%(w/w)) (Figure 1(a) and 1(b)) reflect contribution of molecular entanglement based on their chemical composition. Cellulose content might be one of contributions which affect on viscoelasticity and viscosity properties. According to Yudianti et al. ${ }^{2}$ cellulose content in Salvia hydrogels are 8, 23 and 41\% for SM, SS, SV, respectively, in which SM gel has the lowest cellulose content among these hydrogels. Other contribution is a presence of high arabinose in SM hydrogel which gives a branching and amorphous structure to its main carbohydrate chain.

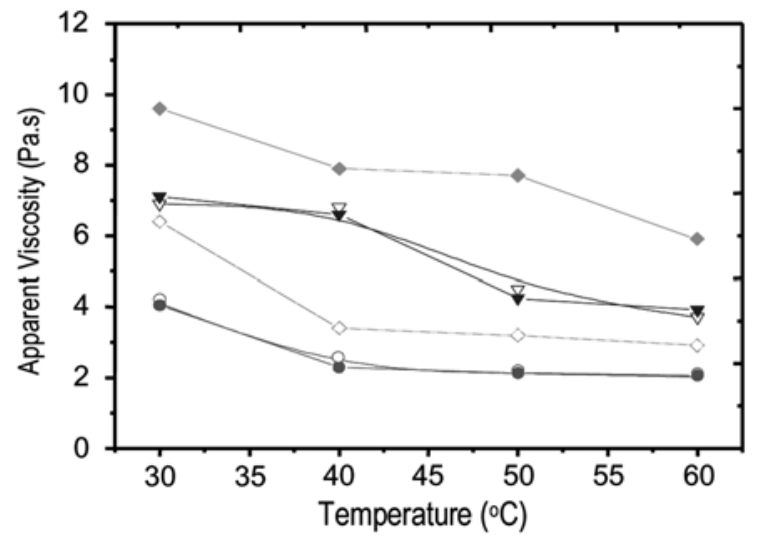

Figure 2. Viscosity properties of salvias hydrogels before (BD) and after drying (AD). ( $\bigcirc$, SM-AD;, SM-BD; $\nabla$, SS-AD; $\nabla$, SS-BD; $\diamond$, SV-AD; $\diamond$, SV-BD).

If cellulose content is low, penetration of water into the whole amorphous region of hydrogel was easy to occur resulting in softening of the hydrogels. Viscosity properties of AD hydrogels from SM and SS were similar to those of BD hydrogels. SV hydrogel which contained the highest cellulose, therefore, formed a hard gel and its viscosity was also the highest (Figure 2). However, viscosity property of AD hydrogel from SV was different from that of its BD hydrogel. If cellulose content is high, cellulose molecules might attach together during drying to form new hydrogen bonds between the molecules resulting in formation of clusters of cellulose rich portions. Thus, high cellulose content gave more crystalline structure to the SV hydrogel and inhibited penetration of water to its amorphous region. It is reasonable that chemical composition and molecular entanglements of the hydrogels determine their rheological properties with cellulose playing an important role to provide hardness to the hydrogels. As a result, since drying changed molecular interactions in the hydrogels, for the further study, BD hydrogel was used to characterize effects of additives on hydrogel formation.

The results of addition of urea to Salvia hydrogels indicate considerable structural change in gel system. All hydrogels showed significant changes from solid (before addition of urea) $\left(G^{\prime}>G^{\prime \prime}\right)$ to liquid-like state (after addition of urea) $\left(G^{\prime \prime}>G^{\prime}\right)$ as shown in Figures 3(a)-(c). Properties of hydrogels are known to be influenced by state of water which contributes in formation of hydrogen bonding network of water molecules and hydrogen bond of water-gel molecules. Addition of urea to gel network changes hydrogen bonding network in gel structure. Urea molecule plays a possible action to make urea-water molecular interactions as investigated by using Energy Calculation and Dynamics (ENCAD) ${ }^{13}$ resulting in disruption of hydrogen bonds in water-gel and water molecules. In the presence of urea, hydrogel forms aqueous gel, suggesting that some or all the hydrogen bonds 


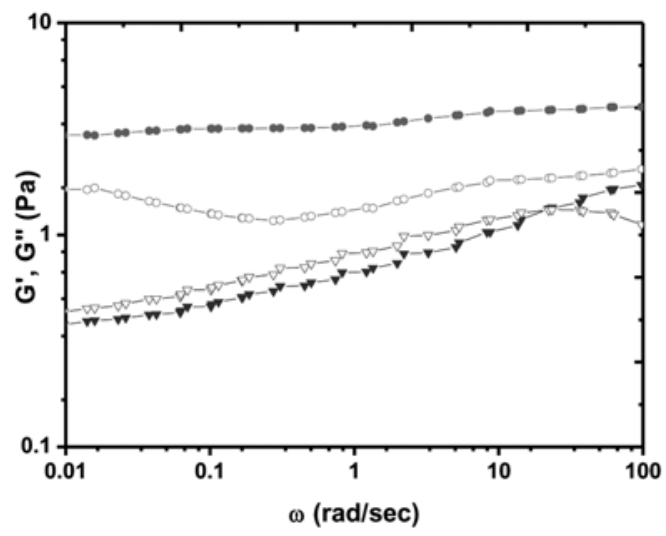

(a)

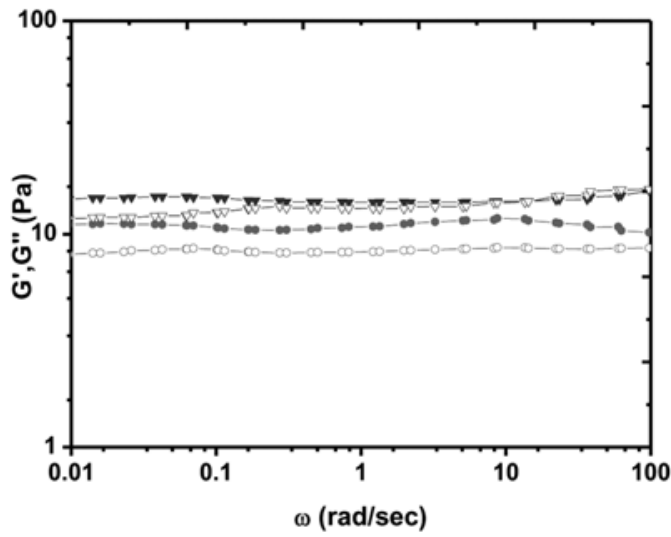

(c)

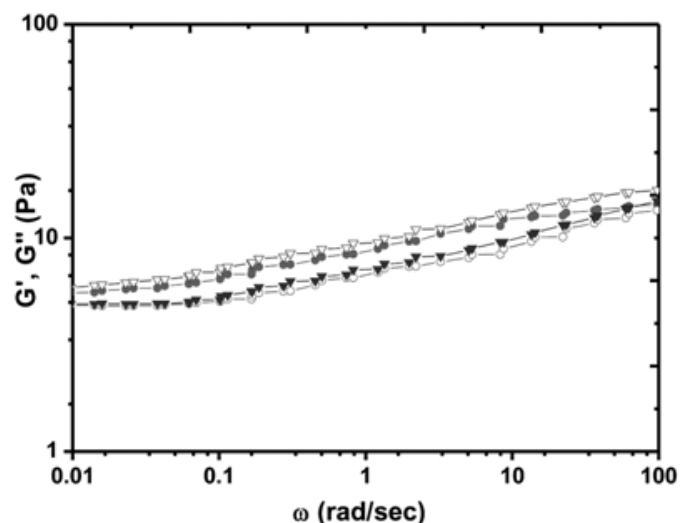

(b)

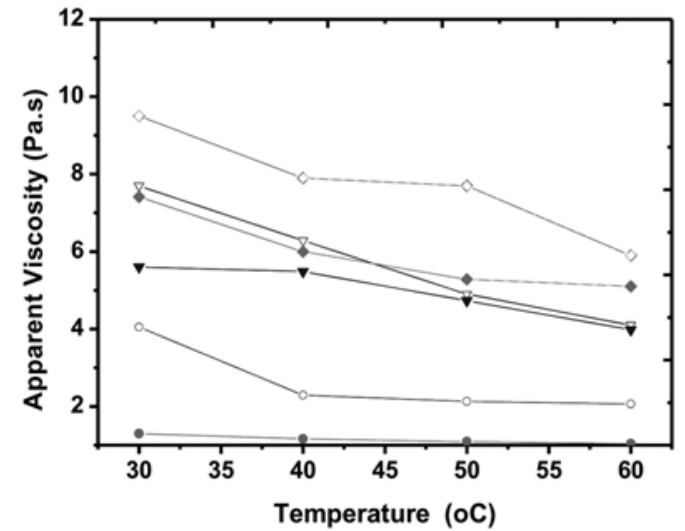

(d)

Figure 3. Effects of urea on viscoelasticity and viscosity properties of Salvia hydrogels. (a) Viscoelasticity properties of SM hydrogel (,$G^{\prime}$, Untreated SM; $\bigcirc, G^{\prime \prime}$, Untreated SM; $\nabla, G^{\prime}$, SM-Urea; $\nabla, G^{\prime \prime}$, SM-Urea), (b) Viscoelasticity properties of SS hydrogel ( $\bullet, G^{\prime}$, Untreated SS; $\bigcirc, G^{\prime \prime}$, Untreated SS; $\boldsymbol{\nabla}, G^{\prime}$, SS-Urea; $\nabla, G^{\prime \prime}$, SS-Urea), (c) Viscoelasticity properties of SV hydrogel ( $\bullet, G$ ', Untreated SV; $\bigcirc$, $G^{\prime \prime}$, Untreated SV; $\nabla, G^{\prime}$, SV-Urea; $\nabla, G^{\prime \prime}$, SV-Urea), and (d) Viscosity properties of Salvia hydrogels (SM, SS and SV) $(\bigcirc$, Untreated SM; ๑, SM-Urea; $\nabla$, Untreated SS; $\boldsymbol{\nabla}$, SS-Urea; $\diamond$, Untreated SV; $\diamond$, SV-Urea).

should be broken. As a consequence, viscosity significantly reduced and conversion from solid to liquid state clearly occurred. The conversion states that hydrogen bonding plays an important role in gel interaction. Importance of hydrogen bonds as one of molecular interactions on gel formation is also shown by reduction of viscosity of the hydrogels after addition of urea at different temperature $\left(30,40,50\right.$ and $\left.60^{\circ} \mathrm{C}\right)$ as shown in Figure 3(d). Action of urea as a hydrogen bond disturbing agent was established in curdlan. ${ }^{14,15}$ Similar results were also verified in the cases on chitosan- $\beta$-glycerolphosphate ${ }^{16}$ and oxalic acid-based chitosan hydrogels. ${ }^{17}$

Other salts such as potassium thiocyanate (KSCN) and potassium sulfate $\left(\mathrm{K}_{2} \mathrm{SO}_{4}\right)$ were added to the hydrogels of $\mathrm{SM}$, SS and SV at concentration of $0.1 \mathrm{M}$, and viscoelastic and viscosity properties were analyzed. Figure 4 showed loss factor $(\tan \delta$ ) and viscosity properties of the SV hydrogel as a representative for Salvia hydrogels. Generally, $0.1 \mathrm{M}$ of $\mathrm{KSCN}$ and $\mathrm{K}_{2} \mathrm{SO}_{4}$ changed the properties of the native gels from gel to more liquid state. In Figure $4, \mathrm{~K}_{2} \mathrm{SO}_{4}$ induced considerable changes in loss factor from solid $(\tan \delta<1)$ (before $\mathrm{K}_{2} \mathrm{SO}_{4}$, addition) to liquid state ( $\tan \delta>1$ ). Contrary to $\mathrm{K}_{2} \mathrm{SO}_{4}$, addition of KSCN to the hydrogels shifted tan $\delta$ to the higher region. Other alternatives to monitor changes of the gel structure were given by viscosity analysis. Addition of $\mathrm{KSCN}$ and $\mathrm{K}_{2} \mathrm{SO}_{4}$ reduced viscosity in arranges of 3-13.6\% and $12.5-62.7 \%$ of the native gel, respectively. Increase of temperature from 30 to $60{ }^{\circ} \mathrm{C}$ also reduced the viscosity from 9.6-7.3 Pa.s at the native state to 9.3-6.6 Pa.s for KSCN and 8.4-2.9 for $\mathrm{K}_{2} \mathrm{SO}_{4}$. Concentration of $0.1 \mathrm{M}$ for KSCN and $\mathrm{K}_{2} \mathrm{SO}_{4}$ are enough to change gel structure of Salvia hydrogels.

Treatment of hydrogels with calcium was intended to know effectiveness of calcium bridge formation in the gel structure. Addition of calcium to hydrogel was conducted after acid treatment. Acid treatment of the native hydrogels increased the amount of protonated carboxyl groups and split out calcium ion bridges between carboxyl groups originally present in the gel structure. As a result, reduction of density of free-charge carboxyl ( $\left.\mathrm{COO}^{-}\right)$diminished electrostatic repulsions and reduction of viscosity and dynamic 


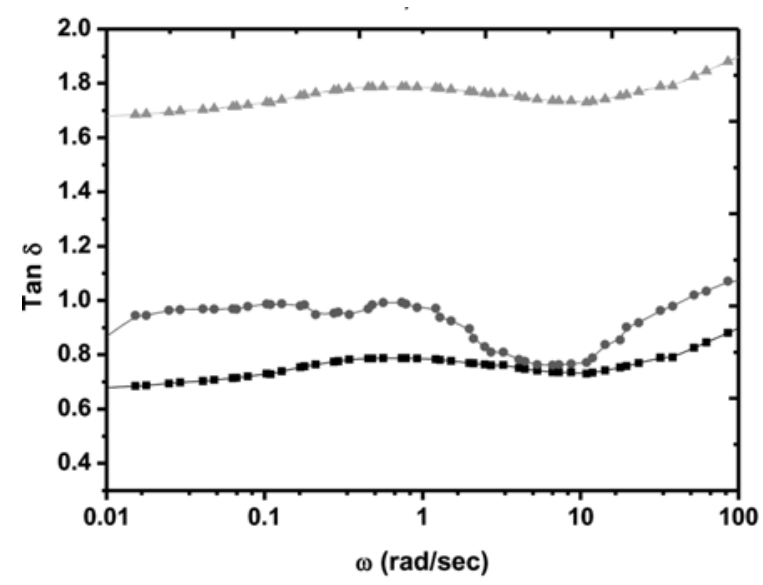

(a)

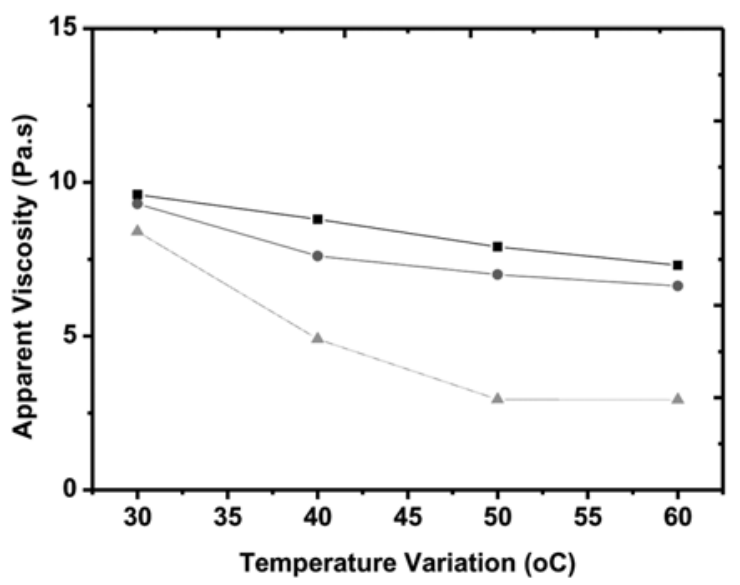

(b)

Figure 4. Effects of addition of $\mathrm{KSCN}$ and $\mathrm{K}_{2} \mathrm{SO}_{4}$ on rheological properties of Salvia hydrogels. (a) Viscoelasticity properties of SV hydrogel ( $\boldsymbol{\square}$, without $\mathrm{KSCN}-\mathrm{K}_{2} \mathrm{SO}_{4} ; \boldsymbol{\bullet}, \mathrm{KSCN} ; \boldsymbol{\Delta}, \mathrm{K}_{2} \mathrm{SO}_{4}$ ) and (b) Viscosity properties of SV hydrogel ( $\boldsymbol{\square}$, without $\mathrm{KSCN}$ $\mathrm{K}_{2} \mathrm{SO}_{4} ; \bullet, \mathrm{KSCN} ; \boldsymbol{\Lambda}, \mathrm{K}_{2} \mathrm{SO}_{4}$ ).

modulus. Concentrations of calcium ion added to the hydrogel were approximately $0.7 \times 10^{-3}, 1.6 \times 10^{-3}$ and $2.8 \times 10^{-3} \%$ $(\mathrm{w} / \mathrm{w})$. Viscoelastic and viscosity properties of the calciumtreated hydrogels were compared with the results from samples of the acid-treated and neutralized hydrogels. The results shown in Figures 5(a) and (b) indicate that the present concentrations were enough to disturb dynamic modulus and viscosity of the hydrogels. Addition of $0.7 \times 10^{-3} \%(\mathrm{w} / \mathrm{w})$ calcium ion to the hydrogels greatly recovered viscosity to 3.2 (SM), 6.5(SS), 10.7(SV) Pa.s closely approached to the viscosity of the native hydrogels (4 (SM), 6.9 (SS), 9.6 (SV) Pa.s). Calcium present in the native Salvia hydrogels at approximately 0.028 (SM), 0.012(SS), 0.01 (SV) mol took a part in function to stabilize network through crosslinking via carboxyl groups of gel molecule. The content of carboxylate in polysaccharide portion of Salvia hydrogels are equivalent to each uronic acid content of 0.065, 0.03, 0.02 mol for respec-

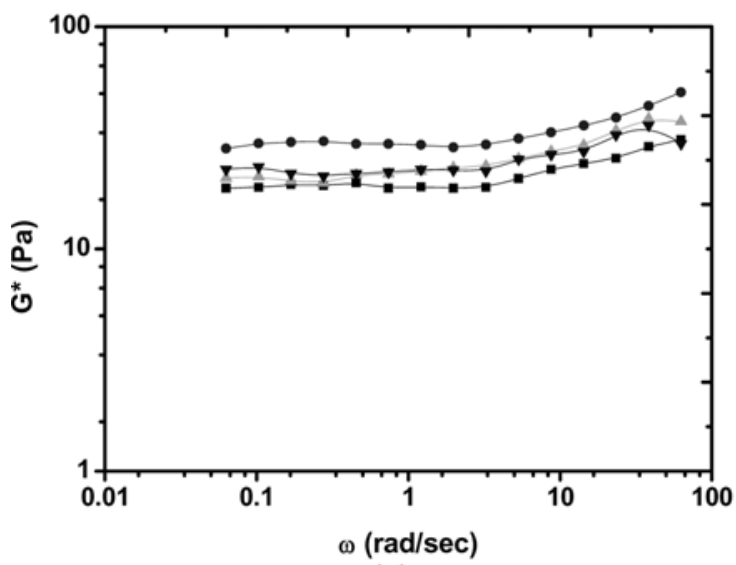

(a)

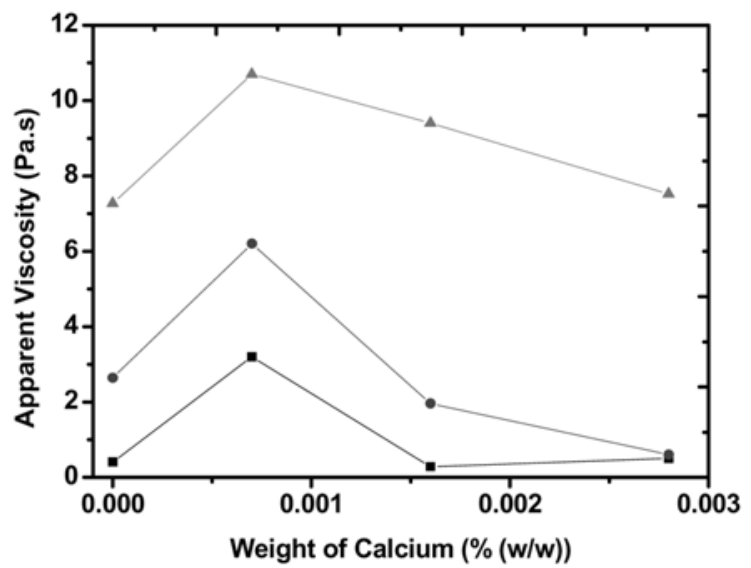

(b)

Figure 5. Effects of calcium on rheological properties of Salvia hydrogels. (a) Viscoelasticity properties of SV hydrogels ( $\mathbf{\square}$, without calcium; . $0.7 \times 10^{-3} \%(\mathrm{w} / \mathrm{w})$ calcium; $\boldsymbol{\Delta}, 1.6 \times 10^{-3} \%(\mathrm{w} / \mathrm{w})$ calcium; $\mathbf{\nabla}, 2.8 \times 10^{-3} \%(\mathrm{w} / \mathrm{w})$ calcium) and (b) Viscosity properties of Salvia hydrogels $(\boldsymbol{\square}, \mathrm{SM} ; \boldsymbol{\mathbf { O }}, \mathrm{SS} ; \boldsymbol{\Delta}, \mathrm{SV})$ in calcium addition of $0.7 \times 10^{-3} \%(\mathrm{w} / \mathrm{w}), 1.6 \times 10^{-3} \%(\mathrm{w} / \mathrm{w}), 2.8 \times 10^{-3} \%(\mathrm{w} / \mathrm{w})$.

tive SM, SS, SV. It was estimated that $80-86 \%$ carboxylate make crosslinkages through calcium ions. Released calcium in acidic condition were probably participated to reform crosslinkage anymore under neutral condition. Although the results indicate that calcium bridges were reformed effectively by addition of $0.7 \times 10^{-3} \%(\mathrm{w} / \mathrm{w})$ calcium ion, addition of a large amout of calcium such as $1.6 \times 10^{-3}$ and $2.8 \times 10^{-3} \%$ (w/w) abruptly reduced viscosity and viscoelastisity of the hydrogel solutions. Similar observations were revealed on rheological properties of acyl gellan after addition of calcium and magnessium. ${ }^{18}$ These calcium concentrations were not predicted to modify new calcium bridges and subsequetly acted as an inhibitor in molecular interaction. We supposed that electrostatic repulsion between free-charged carboxyl groups and calcium crosslinkages simultaneously operated in molecular interaction together with hydrogen bondings. 


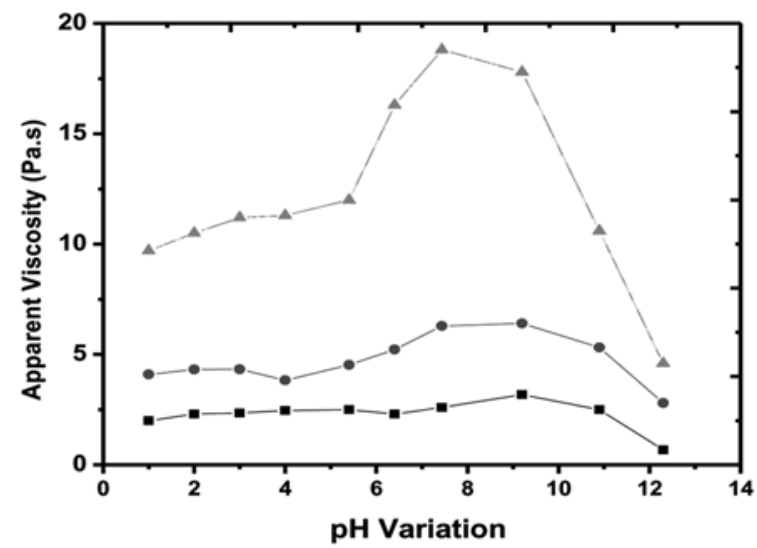

(a)

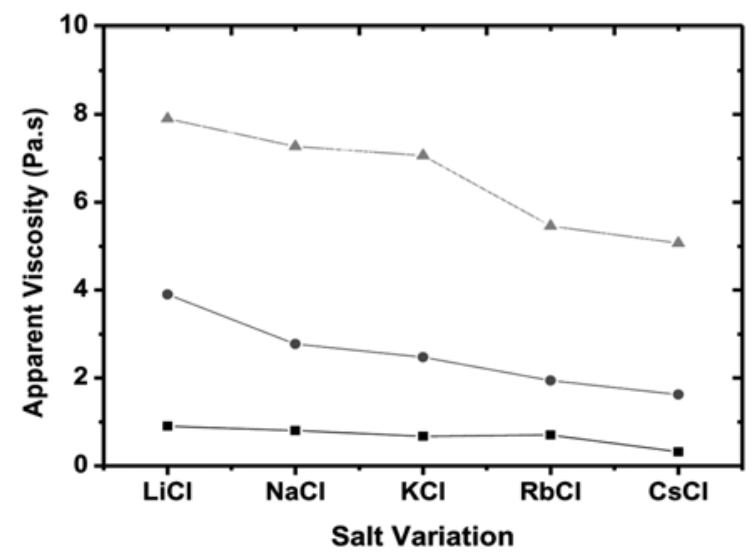

(b)

Figure 6. Effects of $\mathrm{pH}$ and alkali metal salts on viscosity properties of Salvia hydrogels (a) $\mathrm{pH}(\boldsymbol{\square}, \mathrm{SM} ; \boldsymbol{\bullet}, \mathrm{SS} ; \boldsymbol{\Delta}, \mathrm{SV}$ ) and (b) Alkali metal salt ( $\mathbf{\square}, \mathrm{SM} ; \boldsymbol{O}, \mathrm{SS} ; \boldsymbol{\Delta}, \mathrm{SV})$.

Effects of $\mathrm{pH}$ on viscosity properties of the hydrogels were shown in Figure 6(a). Basically, under acidic conditions all Salvia hydrogels had lower viscosities than the values in weak alkaline conditions such as $\mathrm{pH}$ 6-9 with a maximal viscosity value at $\mathrm{pH}$ of 9 and greatly reduced above $\mathrm{pH}$ 9. Under low $\mathrm{pH}$ condition, protonated carboxyl groups caused low viscosity properties as described above, since low viscosity might be caused by decreasing density of negative charges as an origin of electrostatic repulsions. By increasing $\mathrm{pH} 6.5$ to 9 ionized fractions of carboxyl groups increased and almost all carboxyl groups are ionized and present as carboxylate forms ( $\left.\mathrm{COO}^{-}\right)$. It is understandable that under this condition, calcium molecules present at concentration of $0.028(\mathrm{SM}), 0.012(\mathrm{SS}), 0.01$ (SV) mol are perfectly released from the carboxyl groups due to uronic acids to form $\mathrm{COO}^{-}$, disrupting cross-linkages in the gel network. Production of a large amount of $\mathrm{COO}^{-}$may induce additional electrostatic repulsions, thus leading to make high viscoelastic and viscosity properties probably highest at $\mathrm{pH}$ 9. However, under $\mathrm{pH}$ condition above 9, great reduction of viscosity occurred. This behavior may be due to destruction of hydrogen bonds operated both in intra- and inter-molecular associations of the hydrogels and change of molecular conformations into ones which prevent electrostatic repulsions by masking effects of formation of large amount of ONa groups. Destruction of hydrogen bonds under high $\mathrm{pH}$ was familiar in a field of polysaccharide science such as in utilizations of strong alkali for extraction of hemicellulosic polysaccharides from woody materials and production of layon from natural cellulose.

Contribution of electrostatic repulsions was also analyzed by addition of neutral alkali metal chlorides $(\mathrm{LiCl}, \mathrm{NaCl}, \mathrm{KCl}$, $\mathrm{RbCl}$ and $\mathrm{CsCl}$ ) at $0.1 \mathrm{M}$ with changing ion radius from $\mathrm{Li}^{+}$ (smallest radius cation) to $\mathrm{Cs}^{+}$(largest radius ion). Ion-size dependent reduction of viscosity was clearly shown in Figure 6(b). Addition of alkali-metal chlorides as monovalent cations to hydrogel reduced native gel viscosity of 4.0 (SM), 7.7 (SS), 9.5 Pa.s (SV) to nearly 0.3-0.9 (SM), 1.63.9 (SS), 5.1-7.9 Pa.s (SV) depending on size of cations. The viscosity reduced to the lowest values by addition of the largest size cation $\left(\mathrm{Cs}^{+}\right)$. The reduction of viscosity indicated that introduction of large ions which produce longer distances between carboxylate anions diminished electrostatic repulsions between carboxylate groups.

Finally, although the exact increments of three interactions which operated to form hydrogels, electrostatic repulsion, hydrogen bonds and divalent metal ion bridges, cannot be determined. Present results indicate that electrostatic repulsion is a major contributor for production of hydrogel structure.

Acknowledgements. One of authors (R.Y.) was a recipient of RONPAKU Fellow of Japan Society for the Promotion of Science with Program Grant LIPI-10512. This study was also supported in part by a Grant in Aid from the Ministry of Education. Culture, Sports, Science and Technology Japan (to J. A.).

\section{References}

(1) R. Yudianti, L. Indrarti, M. Sakamoto, and J. Azuma, Proceedings of The $5^{\text {th }}$ International Wood Science Symposium, pp. 199-204 (2004).

(2) R. Yudianti, L. Indrarti, M. Sakamoto, and J. Azuma, Proceedings of The $6^{\text {th }}$ International Wood Science Symposium, pp. 273-277 (2005).

(3) R. Yudianti, L. Indrarti, M. Karina, M. Sakamoto, and J. Azuma, Journal of Tropical Wood Science and Technology, 5, 12 (2007).

(4) R. Candrasekaran, A. Radha, and V. G. Thailambal, Carbohydr. Res., 224, 1 (1992).

(5) E. Loizou, J. T. Weisser, A. Dundigalla, and G. Schmidt, Macromol. Biosci., 6, 711 (2006).

(6) M. A. Torres, M. M. Beppu, and E. J. Arruda, Brazilian J. Food Technol., 9, 2, 101 (2006).

(7) J. Tang, J. Lelievre, M. A. Tung, and Y. Zeng, J. Food Sci., 
59, 1 (1994).

(8) J. Tang, M. A. Tung, and Z. Yanyin, Food Sci. J., 60 (1995).

(9) F. Yamamoto and R. L. Cunha, Carbohydr. Polym., 68, 517 (2007).

(10) Rodriguez-Hernandez, S. Durand, C. Garnier, A. Tecante, and J. L. Doublier, Food Hydrocolloid, 17, 621 (2003).

(11) R. Fijan, S. Sostar-Turk, and R. Lapasin, Carbohydr. Polym., 68, 708 (2007).

(12) E. Gregorova, W. Pabst, and J. Stetina, Ceram. Silikaty, 50, 232 (2006).

(13) J. Tsai, M. Gerstein, and M. Levitt, J. Chem. Phys., 104, 9417
(1996).

(14) B. S. Kim, I. D. Jung, J. S. Kim, J.-H. Lee, I. Y. Lee, and K. B. Lee, Biotechnol. Lett., 22, 1127 (2000).

(15) D. W. Renn, Carbohydr. Polym., 33, 219 (1997).

(16) J. Cho, M.-C. Heuzey, A. Begin, and P. Carreau, Carbohydr. Polym., 63, 507 (2006).

(17) H. Melina, C. H. Marie, and B. Andre, Hydrogel. Rheology Acta, 45, 659 (2006).

(18) H. Yiqun, S. Pawan P., T. Juming, and S. Bary G., Carbohydr. Polym., 56, 27 (2004). 